\title{
The Proliferation of End Users as a Success Metric for Cryptocurrencies
}

\author{
Leonardo Maria De Rossi \\ Department of Digitalization \\ Copenhagen Business School \\ Copenhagen, Denmark \\ lmdr.digi@cbs.dk
}

\author{
Michel Avital \\ Department of Digitalization \\ Copenhagen Business School \\ Copenhagen, Denmark \\ michel@avital.net
}

\author{
Rob Gleasure \\ Department of Digitalization \\ Copenhagen Business School \\ Copenhagen, Denmark \\ rg.digi@cbs.dk
}

\begin{abstract}
Over the last decade, numerous studies have examined the remarkable appreciation of cryptocurrencies and have typically focused on their price and the factors that predict them. In contrast, this paper argues that the success of a cryptocurrency is determined not only by its monetary value but also by the proliferation of its end users. Specifically, we hypothesize that changes in developers' and miners' activities drive the growing proliferation of a cryptocurrency's end users. Building on the Bitcoin case, we use a time-series model based on 4,285 Bitcoin daily observations to suggest that changes in the number of end users are anticipated by surges or drops in activity by the developers and miners who develop and maintain the network. We further find a limited relationship between these variables and the price of Bitcoin. These results support an alternative view of cryptocurrencies' success and highlight further research avenues in this nascent domain.
\end{abstract}

\section{Introduction}

Blockchain is frequently referred to as one of the main technological innovations of the $21^{\text {st }}$ century, with the potential to reshape and disrupt a range of economic activities [1]. Blockchain was developed as a supporting technology for the cryptocurrency Bitcoin [2]. More broadly, blockchain has been described as key to the development of the Internet of Value [3], whereby blockchain can provide the foundational technology [4] that might enable a global peer-to-peer value exchange infrastructure.

In the first half of 2021, the cryptocurrencies market has gained global attention due to an unpredictable sequence of events. First, many incumbents, such as Microstrategy, Square and Tesla, have entered the market, acquiring significant amounts of cryptocurrencies (mainly Bitcoin) as a store of value. Second, as a natural consequence of this corporate interest, the market reached its All-Time-
High (ATH) market capitalization of \$2.4T [5], led by the rapid growth of several cryptocurrencies, including Bitcoin, Ethereum, Cardano, and Doge, to name a few. Third, the market faced a sharp decrease after tweets by Elon Musk criticized Bitcoin's energy consumption [6]. Finally, it faced a second drastic decrease after the Chinese government banned Bitcoin [7].

The volatility of the market has fueled doubts, with many experts in the field wondering if cryptocurrencies have any intrinsic value. As just one example, Steven Hanke, an economist at Johns Hopkins University, tweeted on March $13^{\text {th }}, 2021$, "Remember what I have said since day 1: Bitcoin is a highly speculative asset, with a fundamental value of ZERO!"

Many academics have searched for a viable model to justify the value of cryptocurrencies. The initial attempts (e.g., [8]-[11]) have generally developed price-formation models considering either economic or market aspects. Subsequent researchers (e.g., [12]) developed models focused on other aspects, such as the miners' activities. Further, some researchers (e.g., [13], [14]) introduced the idea of correlating the developers' activity with the price of a cryptocurrency. All of these studies have a common line of reasoning: they study cryptocurrencies from the price perspective, with the assumption that price is the only indicator of success.

Inspired by the network effects literature (e.g., [15]), we argue that the success of a cryptocurrency is not represented only by its price but also by the proliferation of its end users. The idea that the success of software technology is represented by the proliferation of its end users comes from the opensource software (OSS) theory; multiple researchers have considered OSS success in terms of the size of the consumer base [16]-[19].

Subsequently, we argue that a successful cryptocurrency emerges from the activities of two communities at its basis: developers and miners. 
Thus, this paper explores the following research question:

\section{What is the effect of developers' and miners' activity on the proliferation of a cryptocurrency's end users?}

To answer this question, we rely on a time-series analysis of a single case of cryptocurrency: Bitcoin.

We selected Bitcoin for two major reasons. First, Bitcoin is the cryptocurrencies market leader, accounting for $40 \%$ of the entire market in June 2021 . Second, a fundamental prerequisite to developing significant time-series analysis is the availability of large amounts of data. As the oldest cryptocurrency, Bitcoin offers a longer timeline to study than any other cryptocurrency in the market.

The study is structured as follows. In section 2, we provide a literature review. In section 3, we describe the collected data. In section 4 , we describe the exploratory and statistical analysis. Finally, in section 5 , we provide some concluding remarks on the basis of the statistical insights.

\section{Literature Review}

Consistent with previous studies, our literature review focuses on Bitcoin research. Other cryptocurrencies have thus far received limited attention except in the case of idiosyncracies in those currencies (e.g., Libra's association with Facebook [20], Monero's focus on privacy [21]).

In this section, we discuss the foundation of Bitcoin, give an overview of existing attempts to develop a value-formation model, and introduce a new approach integrating prior attempts.

\subsection{Bitcoin Foundations}

Bitcoin was the original blockchain protocol and remains the most famous of its technological applications. The first Bitcoin white paper [2] was released on 31 October 2008 on a cryptography mailing list by a person (or group) operating under the pseudonym "Satoshi Nakamoto". Nakamoto claimed to have worked "on a new electronic cash system that's fully peer-to-peer, with no trusted third party".

Two crucial innovations underpin Bitcoin [22]: the public and distributed ledger technology called "blockchain", which securely maintains an immutable record of all user transactions, and the existence of an open network of users called "miners", who lend computing power to secure the network in exchange for newly minted Bitcoins [23], [24].

Bitcoin officially went online on 3 January 2009, with the unveiling of the Genesis Block, the first block of data ever created. Since then, it has been maintained by two communities in collaboration: the communities of developers and miners.

2.1.1 Community of Developers. From a technical point of view, Bitcoin runs on an open-source software program, called "Bitcoin Core", which is supported by ongoing protocol updates [25]. Bitcoin Core is mainly managed by an open community of developers through a page on GitHub, a hosting site where developers can upload program code and work collaboratively to improve it. This way of collaborating resembles the community-based management of other free open-source software developments (F/OSSD) like Linux and Apache.

For Bitcoin, communities of volunteer software developers collaborate in a hierarchical network and self-select into tasks and roles based on expertise and preferences. As with other F/OSSD, the leadership (and the control it brings) is evident in the roles outlined by the community organizational hierarchy. These roles carry titles such as "developer," "module maintainer," and "release manager." Other roles are less formal [26]. The most important tasks developers address in GitHub are: (1) commits, (2) pull requests, and (3) issues. From the official GitHub Glossary, a commit is a "revision" - that is, an individual change to a file (or set of files). Pull requests are proposed changes to a repository submitted by a user and accepted or rejected by a repository's collaborators. Issues are suggested improvements, tasks or questions related to the repository; they can be created by anyone and are moderated by repository collaborators.

Developers without commit rights to a project must "fork" the project, creating a personal copy of the code that they can change freely. They can then submit some or all of the changes to the original project by issuing a pull request. The project owner or another member with commit rights can then merge their changes. Developers can also communicate about code-related actions by submitting a comment on a commit, an issue, or a pull request [27]. This strict and formalized structure of Bitcoin has been termed "senatorial governance" by Parkin [28].

As of May 2021, Bitcoin Core on GitHub registered a total of $\sim 1,890$ commits, $\sim 750$ pull requests and $\sim 1,100$ issues.

2.1.2 Community of Miners. Bitcoin relies on a distributed network [29], which is composed of miners who collect, verify, and update transactions on a shared public ledger that is publicly auditable [22]. The mining process is a specific cryptographic technique required to add new transactions in a shared ledger into "blocks" of information. Mining is a 
lottery-based process that often requires billions of guesses before a suitable verification solution can be found and a miner can add a block in the shared ledger. A miner's probability of being able to provide the "proof-of-work" depends on the computing power the miner controls [22]. The difficulty of the problem miners must solve changes every 2016 blocks, increasing or decreasing on the basis of the average computational power used by the network to mine the previous 2016 block (a value called "hash rate"), to ensure that a new block is mined every $\sim 10$ minutes.

On 30 April 2021, the hash rate of Bitcoin was $\sim 170,000,000 \mathrm{TH} / \mathrm{s}$ (Terahash per second), meaning that every second $170^{\wedge 18}$ combinations are tried in order to solve this cryptographic puzzle.

Miners receive two types of rewards for this job. First, they receive new coins for each new block they generate. Second, they receive transaction fees from the transactions in each new block they generate. The number of newly generated bitcoins received from mining a block decreases every 210,000 blocks. This number started at 50 bitcoins per block in January 2009, then halved to 25 bitcoins per block in November 2012, then halved a second time to 12.5 bitcoins per block in July 2016. Based on this formula, Bitcoin mining rewards will decrease exponentially until approximately the year 2140, when all bitcoins (20.99999998 million) will have been issued.

\subsection{Bitcoin Price Formation Models}

Given its high visibility and intrinsically disruptive nature [30], Bitcoin has attracted a great deal of attention across different academic disciplines [31]. Many academics have aimed at identifying the determinants of Bitcoin's value.

One of the first contributions was by Buchholz et al. [8] that focused on the number of Bitcoins in circulation, the number of daily transactions in Bitcoin, the total daily volume of transactions in Bitcoin, and the number of searches with the query "Bitcoin" derived from Google Trends.

Subsequently, Kristoufek [9] proposed a model to explain the speculative components in the formation of Bitcoin's price. Kristoufek hypothesized that the variation in price could be explained by the investors' interest, which could be determined through two proxies: the number of searches made on Google using the term "Bitcoin" and the number of unique visits to the Bitcoin page of Wikipedia. The findings suggested the value of Bitcoin is based purely on speculation, indicating that value might follow the dynamics of a bubble market, corroborating Buchholz's perspective.

In the same year, Van Wijk [10] studied the relationship between Bitcoin's price and several macroeconomic variables. Van Wijk's model highlighted a relationship between Bitcoin's price and the Dow Jones Index, in both the short and the long term. Findings also suggested a long-term relationship between Bitcoin's price, the Euro/Dollar exchange rate, and the WTI oil price.

Ciaian et al. [11] integrated the results of these studies. Those authors analyzed Bitcoin's price trend in relation to demand/supply functions, investors' interests, and macroeconomic variables. Their results further supported the accumulating body of research on Bitcoin's price.

Subsequently, Hayes [32] hypothesized a connection between Bitcoin's price and its cost of production (i.e., the cost related to the mining process). The findings demonstrated that adding the hash rate to predictive models could explain up to $80 \%$ of the variance in Bitcoin's price. Taking this cost view further, Bouoiyour and Selmi [33] ran multiple models and concluded that, in the long run, Bitcoin's price seemed to be primarily influenced by only one element - the hash rate.

In 2018, Abbatemarco et al. [12] described the costs and revenues that the Bitcoin network supports in order to ensure its operativity. Those authors demonstrated that Bitcoin has a dedicated hardware infrastructure with cost and profit functions comparable to those of a normal production facility.

Next, Trockman et al. [14] investigated the relationship between active communities of developers and the price of some cryptocurrencies. The authors did not identify any compelling evidence for causality between development activity metrics and market cap [13].

\subsection{Proliferation of End Users: A Metric for Bitcoin Success}

The evolution of Bitcoin literature shows a gradual shift from modelling Bitcoin as an asset to modelling it as a network.

Inspired by network effects literature in the software market (as a general reference: [15]), we believe that an additional metric for Bitcoin success might be the proliferation of its end users.

The idea that a product will become more successful as the number of its end users grows is generally accepted by economists (e.g., [34]-[36]). Currencies provide one of the cleanest examples of network effects: the more popular a currency is, the more useful it is, and the more easily it attracts new users. Thus, the proliferation of end users is a factor that should be considered in the case of cryptocurrencies as well. 
Moreover, the idea of considering the number of users in measuring the success of software programs is also confirmed by many academics in the F/OSSD literature. For example, Sen et al. ([19]) introduced the idea of measuring the success of F/OSSD projects with the total number of "subscriber users". As Bitcoin is open-source software, we believe that Sen et al.'s perspective can be applied to this case as well.

In Bitcoin, end users are represented by a specific cryptographic fingerprint, called an address, used in the same way as the beneficiary name on a check [37].

We thus consider the total number of addresses on the Bitcoin system as another metric for Bitcoin's success.

Further, building on the view of Bitcoin as a production network, we hypothesize that the number of end users will vary according to increased or reduced activity in the two main contributing groups:

\section{H1: Developers' activity affects the proliferation of a cryptocurrency's end users.}

\section{H2: Miners' activity affects the proliferation of a cryptocurrency's end users.}

Figure 1 provides a visual representation of the research model.

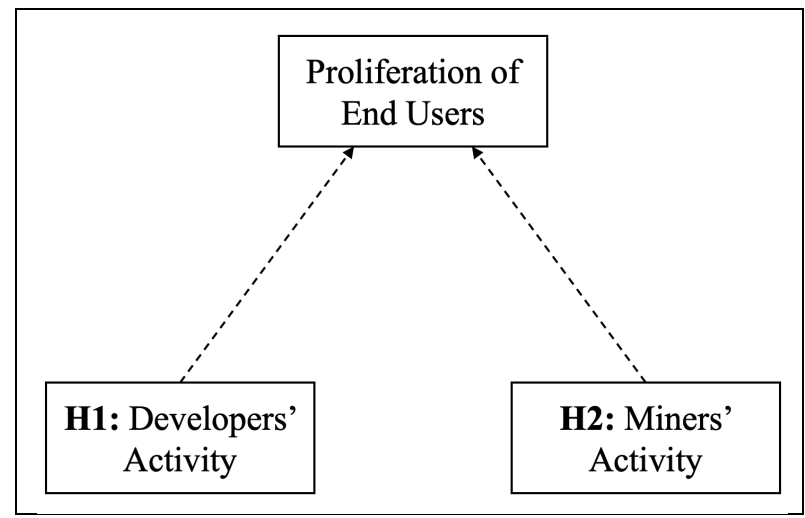

Figure 1. Conceptual research model

\subsection{An Integrated Approach}

To measure the activity of the community of developers, we measure the total number of coding activities on the Bitcoin GitHub page. To measure the activity of the community of miners, we measure the total amount of computing power of the Bitcoin network. Finally, Bitcoin's end users proliferation is measured by the total number of its end users.
Table 1 provides an overview of the variables.

Table 1. Overview of variables

\begin{tabular}{lll}
\hline Variable & Description & Ref \\
\hline $\begin{array}{l}\text { Developers' } \\
\text { Activity }\end{array}$ & $\begin{array}{l}\text { Total amount of } \\
\text { activity on GitHub }\end{array}$ & {$[13],[14]$} \\
\hline $\begin{array}{l}\text { Miners' } \\
\text { Activity }\end{array}$ & $\begin{array}{l}\text { Total amount of } \\
\text { computing power }\end{array}$ & {$[12],[32]$} \\
\hline $\begin{array}{l}\text { Proliferation } \\
\text { of End Users }\end{array}$ & $\begin{array}{l}\text { Number of } \\
\text { end users }\end{array}$ & {$[19],[38]-[40]$} \\
\hline
\end{tabular}

\section{Data Collection}

To answer our research question, we start by collecting daily data about Bitcoin. Specifically, we focus our attention on three main variables: developers' activities, miners' activities and number of end users. We gathered daily observations, beginning on 30 August 2009, the first date for which all the necessary data were available, and ceasing 20 May 2021, for a total of 4,285 observations.

Developers' activity. As mentioned in the literature review, Bitcoin developers mainly collaborate via the Bitcoin page of GitHub.

Inspired by the work of van Tonder et al. [13], we developed a customized script with Python and downloaded the number of daily commits from the Bitcoin Core page on GitHub.

Miners' activity. The computing power of Bitcoin is measured with a value called hash rate. The hashing power is estimated from the number of blocks being mined in the last 24 hours and the current block difficulty. More specifically, given the average time T between mined blocks and a difficulty $\mathrm{D}$, the estimated hash rate per second $\mathrm{H}$ is given by the formula: $H=$ $2^{32} \mathrm{D} / \mathrm{T}$.

The daily hash rate is available from many websites. We selected the website Cryptocompare from which to download the data for two reasons: 1) the availability of a wide range of daily data and 2) the availability of open APIs. Subsequently, we developed a Python script that allowed us to download all the required data.

Proliferation of end users. The proliferation of Bitcoin's end users is measured by the number of active addresses. Active addresses correspond to users who made at least 1 transaction per day. Considering the vast number of "dormant addresses" [41] and to avoid the risk of overestimating the amount of Bitcoin end users, active addresses were considered a more accurate metric for end users. As in the case of the 
aforementioned metrics, we downloaded these data from Cryptocompare.

Table 2 provides an overview of the metrics.

\section{Table 2. Overview of metrics}

\begin{tabular}{lll}
\hline Variable & Metric & Metric Description \\
\hline $\begin{array}{l}\text { Developers' } \\
\text { Activity }\end{array}$ & $\begin{array}{l}\text { Number } \\
\text { of } \\
\text { commits }\end{array}$ & $\begin{array}{l}\text { Number of commits of } \\
\text { GitHub Bitcoin repository }\end{array}$ \\
\hline $\begin{array}{l}\text { Miners' } \\
\text { Activity }\end{array}$ & Hash rate & Hash rate (TeraHash/s) \\
\hline $\begin{array}{l}\text { Proliferation } \\
\text { of End Users }\end{array}$ & $\begin{array}{l}\text { Number active } \\
\text { addresses }\end{array}$ & $\begin{array}{l}\text { Number of addresses with } \\
\text { day } 1 \text { transaction per }\end{array}$ \\
\hline
\end{tabular}

\section{Exploratory Analysis}

Before exploring the presence of any relationships among the aforementioned variables, we conducted some exploratory analyses. All the analyses described in this research paper have been conducted using $\mathrm{R}$ 4.1.0, a free advanced software environment for statistical computing and graphics.

The data used in the analysis are time series data, which constitute a sequence of data points indexed in time order. Before exploring the presence of any relationships among the different time series, we analyzed the data in their original form.

Table 3 provides an overview of the three time series, analyzed by minimum value, maximum value, average value and standard deviation.

\section{Table 3. Time series - general overview}

\begin{tabular}{llll}
\hline Metric & $\begin{array}{l}\text { Active } \\
\text { Addresses }\end{array}$ & Commits & $\begin{array}{l}\text { Hash } \\
\text { Rate }\end{array}$ \\
\hline Min & 0 & 0 & 0.00000942 \\
\hline Max & $1,368,446$ & 61 & $199,064,807$ \\
\hline Average & 375,210 & 8.18 & $23,646,982$ \\
\hline $\begin{array}{l}\text { Standard } \\
\text { Deviation }\end{array}$ & 364,695 & 7.22 & $44,024,151$ \\
\hline
\end{tabular}

By analyzing Table 3, it is possible to gain some insights about the time series.

It is likely that active addresses and hash rate have an incremental evolution over time, while commits do not. This means the difference between the minimum value and the maximum value of the three time series is significant.

Hash rate has the highest difference between min and max. This suggests that the computing power of
Bitcoin value has drastically increased over time. Similar behavior is shown by active addresses, which has increased from 0 to 1.3 million.

Commits have more linear growth, with a reduced standard deviation. To get a visual representation of the three different timeseries, we generated the related plots. Figures 2-4 represents the evolution of active addresses, commits, and hash rate in the selected time range.

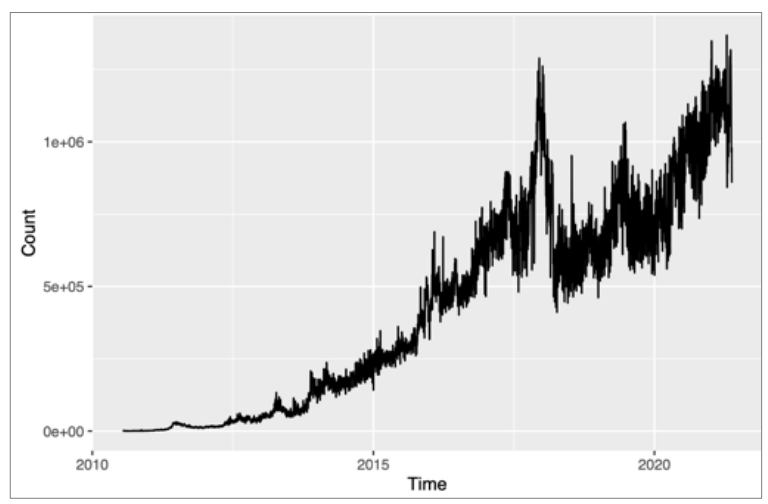

Figure 2. Number of active addresses

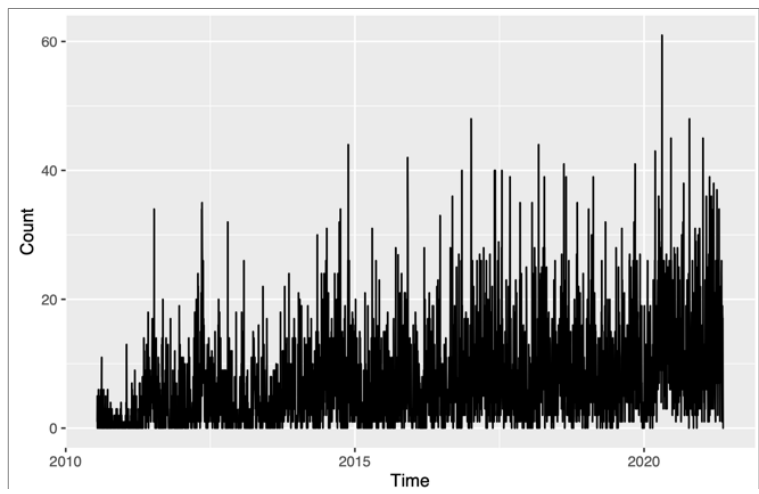

Figure 3. Number of commits

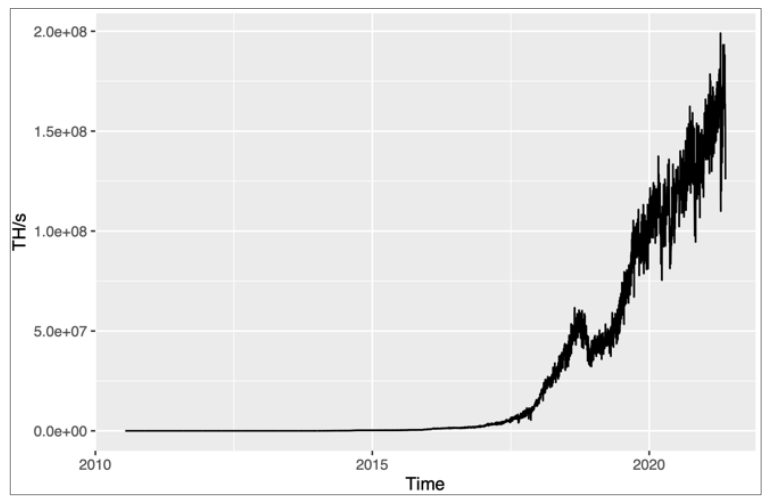

Figure 4. Total hash rate (TH/s)

The three plots confirmed the different trajectories of growth over time. Active addresses show an 
incremental evolution over time, with some peaks (2018 and 2021) followed by some crashes. Commits show a more erratic pattern. Finally, hash rate shows consistent positive growth, again with seemingly erratic changes.

\section{Statistical Analysis}

To test the relationship between active addresses, number of commits and hash rate, we adopted two models: the Vector Auto Regressive (VAR) model and Granger Causality Test (GCT).

VAR is a stochastic process model that is useful to explore whether two time series (A and B) can predict each other.

GCT allows us to understand the order (i.e., direction) of prediction (i.e., $\mathrm{A} \rightarrow \mathrm{B}$ and/or $\mathrm{B} \rightarrow \mathrm{A}$ ).

In our specific case, we are interested in analyzing whether commits and hash rate are useful to forecast active addresses.

\subsection{Preliminary Analysis}

Before applying VAR and GCT, some preliminary analyses of the data are required. In fact, Engle and Granger [42] demonstrated that using these models in the presence of non-stationary variables can lead to spurious results.

Therefore, the first step in the analysis was to determine whether the three series were stationary. We tested for the presence of a unit root through the Augmented Dickey-Fuller (ADF) test. The results suggest that active addresses and commits present stationary time-series ( $\mathrm{p}$-value $<0.01)$, while hash rate is likely non-stationary ( $p$-value $=0.9$ ). In the case of non-stationarity, a typical approach to obtain stationarity is called differencing [43]. Thus, we calculated the first difference of the hash rate timeseries and reexamined the outcome using the ADF stationarity test. The results suggested this value was stationary ( $\mathrm{p}$-value $<0.01)$.

Another crucial test before performing VAR is to determine a suitable number of 'lags' (previous values for each variable). To do this, the Akaike Information Criterion (AIC) was applied. The number of lags selected was 7 for commits and 7 for hash rate.

\subsection{Vector Auto Regressive Model}

In a VAR model, each variable is modeled as a combination of past values of itself and past values of other variables.

Since there is a combination of multiple time series influencing each other, the VAR model is framed as a system of equations with one equation per variable. For example, the system of equations for a VAR (1) model with two time series (variables $Y_{1}$ and $\mathrm{Y}_{2}$ ) is as follows:

$$
\begin{aligned}
& Y_{1, t}=\alpha_{1}+\beta_{11,1} Y_{1, t-1}+\beta_{12,1} Y_{2, t-1}+\varepsilon_{1, t} \\
& Y_{2, t}=\alpha_{2}+\beta_{21,1} Y_{1, t-1}+\beta_{22,1} Y_{2, t-1}+\varepsilon_{1, t}
\end{aligned}
$$

Where, $Y_{1, t-1}$ and $Y_{2, t-1}$ represent the first lag of time series $Y_{1}$ and $Y_{2}$, respectively. The above equation is referred to as a VAR(1) model, because each equation is of order 1 ; that is, it contains up to one lag of each of the predictors $\left(\mathrm{Y}_{1}\right.$ and $\left.\mathrm{Y}_{2}\right)$. In our case, the number of lags is 7 for each model. Moreover, $\mathrm{Y}_{1}$ is always active addresses, while $\mathrm{Y}_{2}$ is commits in Model 1 and hash rate in Model 2.

In the first VAR model, the variance explained with the combination of active addresses and commits was $98 \%$ (Multiple R-Squared $=0.9811$ ). As a result of the iterations between the two time series, 10 out of 14 iterations registered a significant correlation ( $\mathrm{p}$ value $<0.05)$.

In the second VAR model, the variance explained with the combination of active addresses and hash rate was 98\% (Multiple $\mathrm{R}-$ Squared $=0.9817$ ). As a result of the iterations between the two time series, 11 out of 14 iterations registered a significant correlation (p-value $<0.05)$.

\subsection{Granger Causality Test}

The VAR models demonstrated the relationship among the three variables. We next applied the GCT to examine the directionality between variables. First, we applied this function to test our initial hypotheses, $\mathrm{H} 1$ and $\mathrm{H} 2$.

Table 4 shows the result of the two GCT tests.

Table 4. GCT, first results

\begin{tabular}{lll}
\hline Time Series & GCT Results & P-Value \\
\hline $\begin{array}{l}\text { Commits to } \\
\text { Active Addresses }\end{array}$ & Granger-Cause & $<0.0001(* * *)$ \\
\hline $\begin{array}{l}\text { Hash Rate to } \\
\text { Active Addresses }\end{array}$ & Granger-Cause & $<0.0001(* * *)$ \\
\hline
\end{tabular}

From this first analysis, it appears that commits and hash rate affect active addresses. Next, we consider the possibility of bidirectional relationships, which commonly occur in GCT [44]. This means that the active addresses could affect the future of commits and hash rate, and the number of current active addresses could be correlated to the number of future active addresses. 
Table 5 provides an overview of additional tests.

Table 5. GCT, second results

\begin{tabular}{lll}
\hline Time Series & GCT Results & P-Value \\
\hline $\begin{array}{l}\text { Active Addresses } \\
\text { to Commits }\end{array}$ & Granger-Cause & $0.023(*)$ \\
\hline $\begin{array}{l}\text { Active Addresses } \\
\text { to Hash Rate }\end{array}$ & Granger-Cause & $0.012(*)$ \\
\hline $\begin{array}{l}\text { Commits to } \\
\text { Hash Rate }\end{array}$ & No & 0.309 (not sig) \\
\hline $\begin{array}{l}\text { Hash Rate to } \\
\text { Commits }\end{array}$ & No & 0.235 (not sig) \\
\hline
\end{tabular}

The results suggest that changes in active addresses may also drive changes in the number commits and hash rate, although the effect is more uncertain.

Moreover, we wanted to examine whether the newly proposed metric of success is redundant with existing price-based measures of success. If Bitcoin's price is more closely related to its production effort, then the number of active addresses may not be a useful alternative measure. We thus compared the number of active addresses with Bitcoin's trading price. To achieve stationarity in price, we followed the same approach described above (Differencing and ADF Test) and then applied the GCT Test. Table 6 illustrates the results.

Table 6. GCT, third results

\begin{tabular}{lll}
\hline Time Series & GCT Results & P-Value \\
\hline $\begin{array}{l}\text { Price to } \\
\text { Commits }\end{array}$ & No & 0.486 (not sig) \\
\hline $\begin{array}{l}\text { Commits to } \\
\text { Price }\end{array}$ & No & 0.114 (not sig) \\
\hline $\begin{array}{l}\text { Price to } \\
\text { Hash Rate }\end{array}$ & No & 0.087 (not sig) \\
\hline $\begin{array}{l}\text { Hash Rate to } \\
\text { Price }\end{array}$ & Granger-Cause & $0.0207\left(^{*}\right)$ \\
\hline
\end{tabular}

The results identified no relationship of the variables with commits and a possible monodirectional relationship between hash rate and price. These results support a less significant coupling between Bitcoin's price and its network growth, when compared with the number of active addresses.

Finally, the same time-series models were applied to all addresses, as opposed to active addresses only. The results of these models are comparable: VAR models are significant, and the GCT suggests that changes in hash rate and commits affect all addresses as well. These results confirm the validity of our study beyond the initial approximation, indicating robustness to the selection of subsets of addresses.

\section{Discussion}

Blockchain is the cornerstone of a new economic cooperation paradigm characterized by greater decentralization, transparency, security and privacy. This paradigm is aimed at incorporating every trustrelated concept (e.g., governance, accountability and risk) at a technological level, thus enabling the creation of a trustless and secure network without the need for intermediaries [12].

Cryptocurrencies, such as Bitcoin, have attracted the attention of information technology professionals, economists, investors, banks, government, and even the police [45]. The research about cryptocurrency has increased over the years in several disciplines, including business, economy, technology, law, philosophy, and criminology, demonstrating the relevance of this topic [25], [45]. One research topic that has always captured great attention is the value of a cryptocurrency.

Cryptocurrencies are open-source software programs maintained by a community of developers and a community of miners that provide the computing power to maintain the entire network. Therefore, consistent with existing network effects literature, a useful metric for assessing the success of a cryptocurrency is the proliferation of end users who are using the technology. Bank notes, telephones and the Internet have shown that the success of financial systems depends not only on the underlying technologies but also on the acceptance of the systems by large numbers of users.

This study hypothesized that developers' and miners' activity both affect the number of Bitcoin end users. Results supported these hypotheses, providing a statistical model that demonstrates how the number of Bitcoin end users is preceded by two factors: the amount of work that has been done by the developers and the full computing power of the distributed network at its basis.

These findings suggest that, to become successful, a cryptocurrency should exploit an active community of developers dedicated to maintaining the software code at its basis and an extended distributed network of miners running the code (i.e., storing and updating the blockchain, confirming transactions and creating new blocks of information).

We thus find further support for Wang's [46] perspective on F/OSSD survival factors, which highlighted developers' participation and service quality as predictors of survival for both early- and 
late-stage projects. These findings also have more general implications for blockchain.

First, the role of developers suggests that the success of a cryptocurrency may rely heavily on the quality of the code at its core. This conclusion is important because it undermines the contrasting explanations of Bitcoin's success as a 'Greater Fool' Ponzi scheme driven solely by hype (see [47] for more discussion). The focus on technology as opposed to price also warrants some optimism that known issues with cryptocurrencies may receive ongoing attention (e.g., performance, volatility, reliability, unforeseen emerging standards and regulations, and energy cost) [48].

Second, our findings suggest that the network size is vital for the survivability of cryptocurrencies. Some authors have predicted that contemporary cryptocurrencies will give way to newer iterations once the issues are resolved [49]. Others have argued that cryptocurrencies will gradually fall away until they are only used for niche markets or in countries with weak currencies. However, the network effects we observe between miners, developers, and end users undermine those predictions. The Bitcoin community of developers is the most active developer community in the cryptocurrency market, building on more than a decade of developers' and miners' activity. For comparison, Bitcoin Core registered a total of 4,520 commits in 2020, while the second most successful blockchain project (based on both market capitalization and number of users), Ethereum, registered only 780 commits in 2020 on its main GitHub page Go-Ethereum. Even more remarkable is the difference in hash rate. The highest hash rate registered by Bitcoin is $\sim 170,000,000 \mathrm{TH} / \mathrm{s}$, while Ethereum registered a maximum of $\sim 600 \mathrm{TH} / \mathrm{s} .{ }^{1}$

Our study provides both practitioners and researchers with useful insights.

From a research perspective, the model could represent a starting point for further investigating the determinants of the success of cryptocurrencies or blockchain projects in general. First, the model could be tested with other variables, such as pull requests and issues. Second, the presence of network effects in cryptocurrencies should be studied in detail. There are quite a few possible explanations why the number of end users grows when developers and miners become more active. It is conceivable that the effect is causal; for example, it could be an alternative form of herding. Users may be actively watching these supporting groups for cues that the technology is improving. Perhaps the effect is associational, and developers and miners are simply excellent at predicting growth in end-user demand. Another possibility is that all three variables are antecedents of some larger trend. For example, perhaps increasingly prosperous economies are causing all three variables to increase, and we notice the impact on development and infrastructure activities ahead of the effect on price.

As we cannot differentiate between these explanations, future research should explore the reason for the observed network effects. More broadly, future research needs to consider contrasting direct and indirect network effects.

From a managerial perspective, this analysis shows a promising means of distinguishing a successful cryptocurrency from one that is attracting speculative investment. Executives interested in longterm investments in cryptocurrencies may wish to prioritize those projects with an active community of developers and a large network of miners. The aforementioned conclusion assumes a winner-take-all dynamic, whereby a small number of cryptocurrencies will eventually become the dominant players [38].

\section{Limitations and further research}

The contribution of this study should be evaluated in light of some limitations, which also provide directions for future research. First, the study is strictly limited to the case of Bitcoin. Although Bitcoin is the most widely discussed cryptocurrency, it should still be noted that our model has been tested only on Bitcoin data. Therefore, future research could aim at analyzing the robustness of our model to other cryptocurrencies or to other blockchain projects.

Second, other statistical approaches should be tested. Vector Auto Regressions and Granger Causality Tests are popular for testing temporal data, but other alternatives exist that could add further insight. For example, an additional approach could be to apply the Hidden Markov Method, and then relationships among different dependent variables could be observed with Dynamic Linear Regression Models. Quasi-experimental methods such as difference-in-differences testing could also be used to study natural experiments, such as forks or external shocks like high-profile endorsements (or criticisms).

Third, we consider active users as our main metric for Bitcoin's success. However, this metric has three limitations. First, it assumes that Bitcoin is a payment network whose users exchange Bitcoin for services or products. As of today, however, Bitcoin is often considered a store of value rather than a real currency. Eventually, all the addresses could be an alternative metric for success to take into consideration. Second,

\footnotetext{
${ }^{1}$ https://etherscan.io/chart/hashrate
} 
one end-user may own multiple addresses. Likewise, exchanges could hold Bitcoins owned by different end-users in a single address. These factors could serve to make our metric less precise. Finally, the transaction volume has not been considered. Thus, there is no distinction between one user who exchanges one bitcoin and another who exchanges 1000 bitcoins.

\section{Conclusion}

We began this paper by highlighting the importance of identifying the determinants of cryptocurrencies' success beyond the market hype.

For this reason, we created an original database of 4,285 daily data on Bitcoin. Based on this data pool, we developed a time-series model to observe Granger Causality between the success of Bitcoin and the developers' and miners' activities.

Despite the substantial monodirectional emphasis on the price of cryptocurrencies in discourse, we showed that the number of active addresses is a useful alternative metric to study the success of this technology. Finally, we demonstrated that developers' and miners' activities precede an increase in the number of Bitcoin end users.

\section{References}

[1] G. Salviotti, L. M. De Rossi, and N. Abbatemarco, "A structured framework to assess the business application landscape of blockchain technologies.," 2018, doi: 10.24251/hicss.2018.440.

[2] S. Nakamoto, "Bitcoin: a peer-to-peer electronic cash system, October 2008," Cited on, 2008.

[3] A. M. Antonopoulos, The internet of money, vol. 1. Merkle Bloom LLC Columbia, MD, 2016.

[4] M. Iansiti and K. R. Lakhani, "The truth about blockchain," Harvard Business Review, vol. 2017, no. January-February. 2017.

[5] Olga Kharif, "Crypto Market Cap Surpasses \$2 Trillion After Doubling This Year,” Apr. 06, 2021. https://www.bloombergquint.com/globaleconomics/crypto-market-cap-doubles-past-2trillion-after-two-month-surge (accessed May 23, 2021).

[6] Ryan Browne, "Why Elon Musk is worried about bitcoin's environmental impact," $C N B C$, May 13, 2021. https://www.cnbc.com/2021/05/13/whyelon-musk-is-worried-about-bitcoin-

environmental-impact.html (accessed May 23, 2021).

[7] Isabella Weber, "Bitcoin: Why did China crack down on crypto? Fortune," Fortune, May 21, 2021. https://fortune.com/2021/05/21/china-ban- bitcoin-price-bubble-crypto/ (accessed May 23, 2021).

[8] M. Buchholz, J. Delaney, J. Warren, and J. Parker, "Bits and bets: Information, price volatility, and demand for Bitcoin," Econ. 312, 2012.

[9] L. Kristoufek, "BitCoin meets Google Trends and Wikipedia: Quantifying the relationship between phenomena of the Internet era," Sci. Rep., vol. 3, 2013, doi: 10.1038/srep03415.

[10] D. Van Wijk, "What can be expected from the BitCoin," Erasmus Univ. Rotterdam, vol. 18, 2013.

[11] P. Ciaian, M. Rajcaniova, and d'Artis Kancs, "The economics of BitCoin price formation," Appl. Econ., vol. 48, no. 19, pp. 1799-1815, 2016.

[12] N. Abbatemarco, L. M. De Rossi, and G. Salviotti, "An econometric model to estimate the value of a cryptocurrency network. The bitcoin case," 2018.

[13] R. van Tonder, A. Trockman, and C. Le Goues, "A panel data set of cryptocurrency development activity on GitHub," in 2019 IEEE/ACM 16th International Conference on Mining Software Repositories (MSR), 2019, pp. 186-190.

[14] A. Trockman, R. Van Tonder, and B. Vasilescu, "Striking gold in software repositories? An econometric study of cryptocurrencies on GitHub," in IEEE International Working Conference on Mining Software Repositories, 2019, vol. 2019May, doi: 10.1109/MSR.2019.00036.

[15] J. M. Gallaugher and Y. M. Wang, "Understanding network effects in software markets: Evidence from Web server pricing," MIS Quarterly: Management Information Systems, vol. 26, no. 4. 2002, doi: 10.2307/4132311.

[16] J. Feller, B. Fitzgerald, and others, Understanding open source software development. AddisonWesley London, 2002.

[17] K. J. Stewart, A. P. Ammeter, and L. M. Maruping, "A preliminary analysis of the influences of licensing and organizational sponsorship on success in open source projects," 2005, doi: 10.1109/hicss.2005.38.

[18] V. Midha and P. Palvia, "Factors affecting the success of Open Source Software," J. Syst. Softw., vol. 85, no. 4, 2012, doi: 10.1016/j.jss.2011.11.010.

[19] R. Sen, S. S. Singh, and S. Borle, "Open source software success: Measures and analysis," Decis. Support Syst., vol. 52, no. 2, pp. 364-372, 2012.

[20] C. Ye and L. Zhao, "Public Perceptions of Facebook's Libra Digital Currency Initiative: Text Mining on Twitter," 2021, doi: 10.24251/hicss.2021.683.

[21] R. Renwick and R. Gleasure, "Those who control the code control the rules: How different perspectives of privacy are being written into the code of blockchain systems," J. Inf. Technol., vol. 36, no. 1, 2021, doi: 10.1177/0268396220944406. 
[22] Y. Y. Hsieh, J. P. Vergne, P. Anderson, K. Lakhani, and M. Reitzig, "Bitcoin and the rise of decentralized autonomous organizations," $J$. Organ. Des., vol. 7, no. 1, 2018, doi: 10.1186/s41469-018-0038-1.

[23] S. Davidson, P. De Filippi, and J. Potts, "Disrupting Governance: The New Institutional Economics of Distributed Ledger Technology," SSRN Electron. J., 2018, doi: 10.2139/ssrn.2811995.

[24] S. Davidson, P. De Filippi, and J. Potts, "Economics of Blockchain," SSRN Electron. J., 2016, doi: 10.2139/ssrn.2744751.

[25] S. Wang and J. P. Vergne, "Buzz Factor or Innovation Potential: What explains cryptocurrencies' returns?," PLoS One, vol. 12, no. 1, 2017, doi: 10.1371/journal.pone.0169556.

[26] C. Jensen and W. Scacchi, "Collaboration, leadership, control, and conflict negotiation and the Netbeans.org open source software development community," 2005, doi: 10.1109/hicss.2005.147.

[27] L. Dabbish, C. Stuart, J. Tsay, and J. Herbsleb, "Social coding in GitHub: Transparency and collaboration in an open software repository," 2012, doi: 10.1145/2145204.2145396.

[28] J. Parkin, "The senatorial governance of Bitcoin: making (de) centralized money," Econ. Soc., vol. 48, no. 4, pp. 463-487, 2019.

[29] P. Baran, "On Distributed Communications Networks," IEEE Trans. Commun. Syst., vol. 12, no. 1, 1964, doi: 10.1109/TCOM.1964.1088883.

[30] Y. Sompolinsky and A. Zohar, "Secure high-rate transaction processing in bitcoin," in Lecture Notes in Computer Science (including subseries Lecture Notes in Artificial Intelligence and Lecture Notes in Bioinformatics), 2015, vol. 8975, doi: 10.1007/9783-662-47854-7_32.

[31] M. Holub and J. Johnson, "Bitcoin research across disciplines," Inf. Soc., vol. 34, no. 2, 2018, doi: 10.1080/01972243.2017.1414094.

[32] A. S. Hayes, "Cryptocurrency value formation: An empirical study leading to a cost of production model for valuing bitcoin," Telemat. Informatics, vol. 34, no. 7, pp. 1308-1321, 2017.

[33] J. Bouoiyour and R. Selmi, "What does Bitcoin look like?," Ann. Econ. Financ., vol. 16, no. 2, 2015.

[34] J. Farrell and G. Saloner, "Standardization, Compatibility, and Innovation," RAND J. Econ., vol. 16, no. 1, 1985, doi: 10.2307/2555589.

[35] S. S. Oren and S. A. Smith, "Critical Mass and Tariff Structure in Electronic Communications Markets," Bell J. Econ., vol. 12, no. 2, 1981, doi: $10.2307 / 3003567$.

[36] J. Rohlfs, "Theory Of Interdependent Demand For A Communications Service.," Bell J Econ Manag. Sci, vol. 5, no. 1, 1974, doi: 10.2307/3003090.
[37] Andreas M. Antonopoulos, Mastering BitCoin Programming the Open Blockchain, vol. 50, no. 4. 2017.

[38] N. Gandal and H. Halaburda, "Can we predict the winner in a market with network effects? Competition in cryptocurrency market," Games, vol. 7, no. 3, 2016, doi: 10.3390/g7030016.

[39] M. Nair and N. Cachanosky, "Bitcoin and entrepreneurship: breaking the network effect," Rev. Austrian Econ., vol. 30, no. 3, 2017, doi: 10.1007/s11138-016-0348-x.

[40] W. J. Luther, "Cryptocurrencies, Network Effects, And Switching Costs," Contemp. Econ. Policy, vol. 34, no. 3, 2016, doi: 10.1111/coep.12151.

[41] D. Ron and A. Shamir, "Quantitative analysis of the full bitcoin transaction graph," in International Conference on Financial Cryptography and Data Security, 2013, pp. 6-24.

[42] R. F. Engle and C. W. J. Granger, "Co-integration and error correction: Representation, estimation, and testing," Appl. Econom., vol. 39, no. 3, 2015, doi: 10.2307/1913236.

[43] C. W. J. Granger and R. Joyeux, "An Introduction to long-memory time series models and fractional differencing," J. Time Ser. Anal., vol. 1, no. 1, 1980, doi: 10.1111/j.1467-9892.1980.tb00297.x.

[44] C. J. W. Granger, "Investigating Causal Relations by Econometric Models," Econometrica, vol. 37, no. $3,1969$.

[45] G. Molling, A. Klein, N. Hoppen, and R. D. Rosa, "Cryptocurrency: a mine of controversies," JISTEM-Journal Inf. Syst. Technol. Manag., vol. $17,2020$.

[46] J. Wang, "Survival factors for Free Open Source Software projects: A multi-stage perspective," Eur. Manag. J., vol. 30, no. 4, 2012, doi: 10.1016/j.emj.2012.03.001.

[47] A. Blundell-Wignall, "The Bitcoin Question: Currency versus Trust-less Transfer Technology," OECD Work. Pap. Financ. Insur. Priv. Pensions, no. 37, 2014.

[48] P. J. Denning and T. G. Lewis, "Bitcoins Maybe; Blockchains Likely," Am. Sci., vol. 105, no. 6, 2017.

[49] B. Kewell and P. Michael Ward, "Blockchain futures: With or without Bitcoin?," Strateg. Chang., vol. 26, no. 5, 2017, doi: 10.1002/jsc.2149. 